\title{
GREEN CURRENTS FOR QUASI-ALGEBRAICALLY STABLE MEROMORPHIC SELF-MAPS OF $\mathbb{P}^{k}$
}

\author{
VIÊT-ANH NGUYÊN
}

\begin{abstract}
We construct a canonical Green current $T_{f}$ for every quasi-algebraically stable meromorphic self-map $f$ of $\mathbb{P}^{k}$ such that its first dynamical degree $\lambda_{1}(f)$ is a simple root of its characteristic polynomial and that $\lambda_{1}(f)>1$. We establish a functional equation for $T_{f}$ and show that the support of $T_{f}$ is contained in the Julia set, which is thus non empty.
\end{abstract}

2010 Mathematics Subject Classification: Primary: 37F; Secondary: 32U40, $32 \mathrm{H} 50$.

Key words: quasi-algebraically stable meromorphic map, algebraic degree, first dynamical degree, Green current.

\section{Introduction}

Let $f: \mathbb{P}^{k} \rightarrow \mathbb{P}^{k}$ be a meromorphic self-map. Then there are homogeneous polynomials $G_{0}, \ldots, G_{k}$ in the variables $z_{0}, \ldots, z_{k}$ of the same degree $d$ with no nontrivial common factor such that

$$
f=\left[G_{0}: \cdots: G_{k}\right]
$$

in homogeneous coordinates. The polynomial map $F:=\left(G_{0}, \ldots, G_{k}\right)$ is said to be a lifting of $f$ in $\mathbb{C}^{k+1}$. The number $\mathrm{d}(f):=d$ is called the algebraic degree of $f$. Moreover, $f$ is said to be dominant if its Jacobian determinant does not vanish identically (in any local chart). In this work we always consider dominant meromorphic self-maps $f$ of $\mathbb{P}^{k}$ with $k \geq 2$. For $n \in \mathbb{N}, f^{n}$ denotes $f \circ \cdots \circ f$ ( $n$ times). The Fatou set of $f$ is the largest open subset of $\mathbb{P}^{k}$ on which $\left(f^{n}\right)_{n=1}^{\infty}$ forms a normal family. The Julia set of $f$ is, by definition, the complement of its Fatou set in $\mathbb{P}^{k}$.

Recall the following definition (see $[\mathbf{1 5}],[\mathbf{1 6}],[\mathbf{2 0}]$ ):

Definition 1. A meromorphic self-map $f: \mathbb{P}^{k} \rightarrow \mathbb{P}^{k}$ is said to be algebraically stable (or $A S$ for short) if $\mathrm{d}\left(f^{n}\right)=\mathrm{d}(f)^{n}, n \in \mathbb{N}$. 
In other words, $f$ is $\mathrm{AS}$ if and only if a sequence $\left(F_{n}\right)_{n=1}^{\infty}$ of liftings of $\left(f^{n}\right)_{n=1}^{\infty}$ can be defined as follows

$$
F_{n}:=F_{1} \circ F_{n-1}, \quad n \geq 1,
$$

where $F_{1}, F_{0}$ are arbitrarily fixed liftings of $f, f^{0}:=$ Id respectively.

For every AS map $f$ with $\mathrm{d}(f)>1$, N. Sibony proves in $[\mathbf{2 0}]$ that the following limit in the sense of current

$$
T:=\lim _{n \rightarrow \infty} \frac{\left(f^{n}\right)^{*} \omega}{\mathrm{d}\left(f^{n}\right)}
$$

exists, where $\omega$ denotes the Fubini-Study Kähler form on $\mathbb{P}^{k}$ so normalized that $\int_{\mathbb{P} k} \omega^{k}=1$. $T$ is called the Green current associated to $f$. He also proves that $T$ does not charge any hypersurfaces. Given a positive integer $d$, a "generic" meromorphic self-map of algebraic degree $d$ is always AS (see [16]). In the last decades the study of Green currents plays a central role in Complex Dynamics in higher dimensions. We address the reader to the survey articles of Fornæss-Sibony [15], [16], N. Sibony [20], Dinh-Sibony [12] for further explanations. Some other articles on the topic are $[\mathbf{8}],[\mathbf{1 0}],[\mathbf{7}],[\mathbf{1 7}]$.

In contrast to the case of AS maps, the dynamics of non AS maps are, at the moment, still very poorly understood although there has been a lot of activity around this topic in the past few years. Two fundamental problems arise:

Problem 1. Study the degree-growth of non AS maps.

Problem 2. Define a natural Green currents for such maps.

One of the first works in this direction is the article of BonifantFornæss [4] where some special non AS maps are thoroughly studied. In her thesis [3] A. M. Bonifant constructs an appropriate Green current for these maps and then writes down the functional equation. J. Diller and Ch. Favre (see [6]) have constructed Green currents for birational maps of compact Kähler surface. In the case of polynomial maps of $\mathbb{C}^{2}$, the two problems above have been solved by Ch. Favre and M. Jonsson (see [13], [14]). Moreover, S. Boucksom, Ch. Favre and M. Jonsson have investigated the degree-growth for meromorphic surface maps (see [5]). In higher dimension the situation is completely open, and the two questions above remain a great mystery. B. Hasselblatt and J. Propp have studied the degree-growth of monomial maps in [18]. There are series of interesting examples of birational maps acting on the space of complex square matrices which were worked out by E. Bedford and K. Kim and 
others (see $[\mathbf{1}],[\mathbf{2}])$. However, a general theoretical approach still does not exist at the moment.

In this paper we study a new class of non AS self-maps of $\mathbb{P}^{k}$. Under a mild extra assumption on such maps we construct the good Green currents for them, write down the functional equations and show that the support of the Green current is contained in the Julia set of the corresponding self-map. Here is the formal definition of the new class.

Definition 2. A meromorphic self-map $f$ of $\mathbb{P}^{k}$ is said to be quasialgebraically stable (or $Q A S$ for short) if a sequence of liftings $\left(F_{n}\right)_{n=1}^{\infty}$ for the iterates $\left(f^{n}\right)_{n=1}^{\infty}$ can be defined as follows

$$
F_{n}:= \begin{cases}F_{1} \circ F_{n-1}, & n=1, \ldots, n_{0}, \\ \frac{F_{1} \circ F_{n-1}}{H \circ F_{n-n_{0}-1}}, & n>n_{0} .\end{cases}
$$

Here $n_{0} \geq 1$ is an integer, $H$ is a homogeneous polynomial of $k+1$ variables, and $F_{0}:=$ Id and $F_{1}$ is an arbitrarily fixed lifting of $f$.

It is worthy comparing the above recurrent formula with (1.1). In the previous work [19] the author has introduced a criterion in order to test if a non AS self-map is QAS (see conditions (i)-(iii) in Theorem 4.4 below). In fact, in [19] all self-maps which satisfy the latter criterion were called QAS. But in the present work we choose Definition 2 as the new definition for QAS maps. Although this will enlarge the class of QAS self-maps we emphasize that our main objective is to construct canonical Green currents and to study their properties. As it was shown in $[\mathbf{1 9}]$ there are a lot of non AS self-maps which are QAS. In the present work a new family of QAS self-maps in $\mathbb{P}^{2}$ is exhibited.

This paper is organized as follows.

We begin Section 2 by collecting some background and introducing some notation, in order to prepare for the statement of the Main Theorem.

Section 3 is devoted to the proof of the Main Theorem.

Finally, Section 4 concludes the paper with a new family of QAS self-maps in $\mathbb{P}^{2}$.

Acknowledgments. The paper was written while the author was visiting the Université Pierre et Marie Curie and the Korea Institute for Advanced Study (KIAS). He wishes to express his gratitude to these organizations. The first version of this article circulated in a form of a preprint in 2002. 


\section{Statement of the main result}

First we fix some notation and terminology.

2.1. Meromorphic self-maps and positive closed currents of bidegree $(\mathbf{1}, \mathbf{1})$. Let $f$ be a meromorphic self-map of $\mathbb{P}^{k}$. The indeterminacy locus $\mathcal{I}(f)$ of $f$ is the set of all points of $\mathbb{P}^{k}$ where $f$ is not continuous, in other words, the common zero set of component polynomials $G_{0}, \ldots, G_{k}$, where $\left(G_{0}, \ldots, G_{k}\right)$ is a lifting of $f$. So $\mathcal{I}(f)$ is a subvariety of codimension at least 2. The first dynamical degree of $f$, denoted by $\lambda_{1}(f)$, is given by

$$
\lambda_{1}(f):=\lim _{n \rightarrow \infty} \mathrm{d}\left(f^{n}\right)^{\frac{1}{n}} .
$$

For a discussion on dynamical degrees of meromorphic self-maps see the articles of Dinh-Sibony [9], [11]. We denote by $\mathcal{C}^{+}\left(\mathbb{P}^{k}\right)$ the set of positive closed currents of bidegree $(1,1)$ on $\mathbb{P}^{k}$. The mass of $T$ is defined by $\|T\|:=\int_{\mathbb{P}^{k}} T \wedge \omega^{k-1}$. We consider the cone $\mathcal{P}$ of plurisubharmonic functions $u$ in $\mathbb{C}^{k+1}$, satisfying the following homogeneity property: there exist $c>0$ such that if $\lambda \in \mathbb{C}$, then

$$
u(\lambda z)=c \log |\lambda|+u(z), \quad z \in \mathbb{C}^{k+1} .
$$

The functions in $\mathcal{P}$ are so normalized that $\sup u=0$, where $\mathbb{B}$ denotes the unit ball in $\mathbb{C}^{k+1}$.

With a function $u$ satisfying the above homogeneity property (but not necessarily the normalization condition), we are going to associate a current $T \in \mathcal{C}^{+}\left(\mathbb{P}^{k}\right)$. Let $\pi: \mathbb{C}^{k+1} \backslash\{0\} \rightarrow \mathbb{P}^{k}$ be the canonical projection. Let $U$ be an open set in $\mathbb{P}^{k}$ such that there is a holomorphic inverse $s: U \rightarrow \mathbb{C}^{k+1} \backslash\{0\}$ of $\pi$, that is, $\pi \circ s=\mathrm{Id}$. Define $T$ on $U$ by $T:=$ $\operatorname{dd}^{\mathrm{c}}(u \circ s)$. Then $T$ is independent of $s$. With this local definition, we have an operator $\mathcal{L}$ defined by $\mathcal{L}(u):=T$. It is well-known (see [15], [20]) that $\mathcal{L}$ is an isomorphism between $\mathcal{P}$ and $\mathcal{C}^{+}\left(\mathbb{P}^{k}\right)$. If $T=\mathcal{L}(u)$, then we say that a function of the form $u+c$ is a potential of $T$, where $c \in \mathbb{R}$ is a constant. Given a potential $u$ of a current $T \in \mathcal{C}^{+}\left(\mathbb{P}^{k}\right)$, we have that $u(\lambda z)=\|T\| \cdot \log |\lambda|+u(z)$ for $z \in \mathbb{C}^{k+1}, \lambda \in \mathbb{C}$.

Any (not necessarily reduced) hypersurface $\mathcal{H}$ of $\mathbb{P}^{k}$ defines a current of integration $[\mathcal{H}]=[H=0] \in \mathcal{C}_{1}^{+}\left(\mathbb{P}^{k}\right)$, where $H: \mathbb{C}^{k+1} \rightarrow \mathbb{C}$ is a homogeneous polynomial defining $\mathcal{H}$. Moreover,

$$
\|[H=0]\|=\operatorname{deg}(H),
$$

where $\operatorname{deg}(H)$ is the (homogeneous) degree of $H$. 
For a current $T \in \mathcal{C}^{+}\left(\mathbb{P}^{k}\right)$, we define the pull-back of $T$ by $f$ as follows

$$
f^{*} T:=\mathcal{L}(u \circ F),
$$

where $u:=\mathcal{L}^{-1}(T)$ and $F$ is a lifting of $f$. It is easy to see that

$$
\left\|f^{*} T\right\|=\mathrm{d}(f) \cdot\|T\| .
$$

Finally, for a function $u: \mathbb{C}^{k+1} \rightarrow[-\infty, \infty]$, let $u^{*}$ denote its upper semicontinuous regularization, that is, $u^{*}(z):=\lim _{\sup _{w \rightarrow z}} u(w), z \in$ $\mathbb{C}^{k+1}$.

2.2. Statement of the main result. Let $f$ be a QAS self-map as in Definition 2. To simplify the notation, from now on we will write $\lambda$ for $\lambda_{1}(f)$ and $h$ for $\operatorname{deg}(H)$. As an immediate consequence of Definition 2, we have that

$$
\mathrm{d}\left(f^{n}\right)= \begin{cases}\mathrm{d}(f)^{n}, & n=0, \ldots, n_{0}, \\ \mathrm{~d}(f) \cdot \mathrm{d}\left(f^{n-1}\right)-h \cdot \mathrm{d}\left(f^{n-n_{0}-1}\right), & n>n_{0} .\end{cases}
$$

On the other hand, it has been shown in [19, Theorem 4.2] that $\lambda$ is the root of maximal modulus of the so-called characteristic polynomial of $f$

$$
P(t)=t^{n_{0}+1}-d t^{n_{0}}+h .
$$

Now we are ready to formulate the following

Main Theorem. Under the above hypothesis and notation, suppose in addition that $\lambda$ is a simple root of $P(t)$ and $\lambda>1$.

(i) Then $\left(\limsup _{n \rightarrow \infty} \frac{\log \left\|F_{n}\right\|}{\mathrm{d}\left(f^{n}\right)}\right)^{*}$ exists and defines a plurisubharmonic function $u$ in $\mathbb{C}^{k+1}$.

(ii) Let $T:=\mathcal{L}(u)$. Then the following functional equation holds

$$
f^{*}(T)=\lambda \cdot T+\frac{d-\lambda}{h} \cdot[H=0] .
$$

(iii) The support of the current $T$ is contained in the Julia set of $f$, which is thus non empty.

It is worthy to remark here that the presence of a factor $[H=0]$ in the functional equation in (ii) characterizes QAS self-maps. Indeed, for an AS map $f$ with corresponding Green current $T$ (see (1.2)), the functional equation is $f^{*} T=\mathrm{d}(f) \cdot T$.

We know from [19, Theorem 4.2] that the multiplicity of $\lambda$ is either 1 or 2. It is equal to 2 only if $h=\left(\frac{d}{n_{0}+1}\right)^{n_{0}+1} n_{0}^{n_{0}}$. Moreover, it is clear by (2.5) that $\lambda>1$ if and only if $h>1$. 
The author does not know if the QAS self-maps considered in this work can be made algebraically stable by blowing up the projective space, in which case the existence of invariant currents is already known in general. However, the author thinks that the idea of QAS self-maps and the technique presented here could be extended to a more general context, for example, meromorphic self-maps on compact Kähler manifolds.

\section{Proof}

In this section we keep the hypothesis and notation introduced just before the Main Theorem in Subsection 2.2.

Lemma 3.1. Let $r$ be the multiplicity of $\lambda$ in $P(t)$.

1) There exist $Q \in \mathbb{R}[t]$ with $\operatorname{deg} Q=r-1$ and $0<\rho<1$ such that

$$
\mathrm{d}\left(f^{n}\right)=\lambda^{n}\left(Q(n)+\mathcal{O}\left(\rho^{n}\right)\right) .
$$

2) There exists a finite positive constant $C$ such that for all $n \in \mathbb{N}$,

$$
\frac{\mathrm{d}\left(f^{n+1}\right)-\lambda \mathrm{d}\left(f^{n}\right)}{\mathrm{d}\left(f^{n}\right)} \leq \frac{C}{n} \quad \text { and } \quad \sum_{j=0}^{n} \mathrm{~d}\left(f^{j}\right) \leq C \mathrm{~d}\left(f^{n}\right) .
$$

Proof: To prove Part 1) we proceed as in the proof of Theorem 4.2 in [19]. Let $\lambda_{1}, \ldots, \lambda_{n_{0}+1}$ be all the roots of $P$ counted with multiplicities. There are two cases to consider.

Case 1: all roots of $P$ are distinct.

By the proof of Case 1 in Theorem 4.2 in $[\mathbf{1 9}]$, there exist $c_{j} \in \mathbb{C}$ for $j=1, \ldots, n_{0}+1$ such that

$$
\mathrm{d}\left(f^{n}\right)=\sum_{j=1}^{n_{0}+1} c_{j} \lambda_{j}^{n}, \quad n \in \mathbb{N} .
$$

Moreover, if

$$
\left|\lambda_{1}\right|=\max _{c_{j} \neq 0, j=1, \ldots, n_{0}+1}\left|\lambda_{j}\right|,
$$

then $\lambda_{1}>0$ and $c_{1} \in \mathbb{R}$. This implies Part 1) for $\lambda:=\lambda_{1}$ and $Q(t):=c_{1}$.

Case 2: $P$ has a multiple root.

Recall from Case 2 in the proof of Theorem 4.2 in [19] that $h=$ $\left(\frac{d}{n_{0}+1}\right)^{n_{0}+1} n_{0}^{n_{0}}$ and that the only multiple root of $P$ is $\lambda_{n_{0}}:=\frac{d n_{0}}{n_{0}+1}$ which is, in fact, a double root. We may assume without loss of generality that 
$\lambda_{n_{0}+1}=\lambda_{n_{0}}$. Moreover, there exist $c_{j} \in \mathbb{C}$ for $j=1, \ldots, n_{0}-1$, and $c_{n_{0}}, c_{n_{0}+1} \in \mathbb{R}$ such that

$$
\mathrm{d}\left(f^{n}\right)=\sum_{j=1}^{n_{0}-1} c_{j} \lambda_{j}^{n}+\left(n c_{n_{0}}+c_{n_{0}+1}\right) \lambda_{n_{0}}^{n}, \quad n \in \mathbb{N} .
$$

Let

$$
\mu:=\max _{c_{j} \neq 0, j=1, \ldots, n_{0}+1}\left|\lambda_{j}\right|
$$

There are two subcases to consider.

Case 2a: $\mu \neq \lambda_{n_{0}}$.

Then $\mu>\lambda_{n_{0}}$. We argue as in Case 1 using (3.2) instead of (3.1). Consequently, if $\left|\lambda_{1}\right|=\mu$ then we can show that $\lambda_{1}>0$ and $c_{1} \in \mathbb{R}$. This proves Part 1) for $\lambda:=\lambda_{1}$ and $Q(t):=c_{1}$.

Case 2b: $\mu=\lambda_{n_{0}}$.

By (3.2) we see that Part 1) holds for $\lambda:=\lambda_{n_{0}}$ and $Q(t):=c_{n_{0}} t+$ $c_{n_{0}+1}$. This completes the proof of Part 1).

Part 2) is an immediate consequence of Part 1).

In this section we make the following convention: $\mathrm{d}\left(f^{n}\right)=0$ for all $n<0$.

Lemma 3.2. The following identity holds

$$
F_{n}= \begin{cases}F_{n-1} \circ F, & n=1, \ldots, n_{0}, \\ \frac{F_{n-1} \circ F}{H^{\mathrm{d}\left(f^{n-n_{0}-1}\right)},} & n>n_{0} .\end{cases}
$$

Moreover for all currents $T \in \mathcal{C}^{+}\left(\mathbb{P}^{k}\right)$,

$$
\left(f^{n}\right)^{*} T= \begin{cases}f^{*}\left(\left(f^{n-1}\right)^{*} T\right), & n=1, \ldots, n_{0} \\ f^{*}\left(\left(f^{n-1}\right)^{*} T\right)-\|T\| \cdot \mathrm{d}\left(f^{n-n_{0}-1}\right) \cdot[H=0], & n>n_{0}\end{cases}
$$

Proof: We only need to prove identity (3.3) since identity (3.4) is an immediate consequence of (3.3) using (2.2)-(2.3). Observe that by the hypothesis on $f,(3.3)$ is true for $n=1, \ldots, n_{0}$. Supposing (3.3) true for $n$, we need to prove it for $n+1$. 
We have that

$$
\begin{aligned}
F_{n} \circ F & =\frac{F \circ F_{n-1} \circ F}{H \circ F_{n-n_{0}-1} \circ F}=\frac{F\left(F_{n-1} \circ F\right)}{H\left(F_{n-n_{0}-1} \circ F\right)} \\
& =\frac{F\left(H^{\mathrm{d}\left(f^{n-n_{0}-1}\right)} F_{n}\right)}{H\left(H^{\mathrm{d}\left(f^{n-2 n_{0}-1}\right)} \cdot F_{n-n_{0}}\right)}=\frac{H^{\mathrm{d}(f) \cdot \mathrm{d}\left(f^{n-n_{0}-1}\right)} \cdot F \circ F_{n}}{H^{h \cdot \mathrm{d}\left(f^{n-2 n_{0}-1}\right)} \cdot H \circ F_{n-n_{0}}} \\
& =H^{\mathrm{d}(f) \cdot \mathrm{d}\left(f^{n-n_{0}-1}\right)-h \cdot \mathrm{d}\left(f^{n-2 n_{0}-1}\right)} F_{n+1}=H^{\mathrm{d}\left(f^{n-n_{0}}\right)} F_{n+1},
\end{aligned}
$$

where the first equality follows from Definition 2, the third one from the hypothesis of induction, and the last one from identity (2.4). Hence, (3.3) is true for $n+1$.

$$
\text { Put, for } N \geq 1 \text {, }
$$

$$
\sigma_{N}:=\frac{1}{N} \sum_{n=0}^{N-1} \frac{1}{\mathrm{~d}\left(f^{n}\right)}\left(f^{n}\right)^{*} \omega .
$$

Then $\left(\sigma_{N}\right)$ is a sequence of positive closed currents of bidegree $(1,1)$ such that $\left\|\sigma_{N}\right\|=1$. Therefore, we can extract a convergent subsequence $\left(\sigma_{N_{j}}\right): \sigma_{N_{j}} \rightarrow \sigma$. Here, $\sigma$ is a positive closed currents of bidegree $(1,1)$ such that $\|\sigma\|=1$.

Lemma 3.3. The following functional equation holds

$$
f^{*} \sigma=\lambda \cdot \sigma+\frac{d-\lambda}{h} \cdot[H] .
$$

Proof: We have that

$$
\begin{aligned}
f^{*} \sigma_{N}-\lambda \sigma_{N}= & \frac{1}{N} \sum_{n=0}^{N-1}\left(\frac{f^{*}\left(\left(f^{n}\right)^{*} \omega\right)}{\mathrm{d}\left(f^{n}\right)}-\frac{\lambda\left(f^{n+1}\right)^{*} \omega}{\mathrm{d}\left(f^{n+1}\right)}\right) \\
& +\frac{1}{N}\left(\lambda \frac{\left(f^{N}\right)^{*} \omega}{\mathrm{d}\left(f^{N}\right)}-\lambda \omega\right) \\
= & \frac{1}{N} \sum_{n=0}^{N-1} \frac{f^{*}\left(f^{n}\right)^{*} \omega-\left(f^{n+1}\right)^{*} \omega}{\mathrm{d}\left(f^{n}\right)} \\
& +\left(\frac{1}{N} \sum_{n=0}^{N-1} \frac{\mathrm{d}\left(f^{n+1}\right)-\lambda \mathrm{d}\left(f^{n}\right)}{\mathrm{d}\left(f^{n}\right)} \cdot \frac{\left(f^{n+1}\right)^{*} \omega}{\mathrm{d}\left(f^{n+1}\right)}+\frac{\lambda}{N}\left(\frac{\left(f^{N}\right)^{*} \omega}{\mathrm{d}\left(f^{N}\right)}-\omega\right)\right) \\
:= & I+I I .
\end{aligned}
$$


Applying Lemma 3.2 yields that

$$
I=\left(\frac{1}{N} \sum_{n=0}^{N-1} \frac{\mathrm{d}\left(f^{n-n_{0}}\right)}{\mathrm{d}\left(f^{n}\right)}\right)[H] .
$$

Recall from (2.3) that $\left\|\frac{\left(f^{n}\right)^{*} \omega}{\mathrm{d}\left(f^{n}\right)}\right\|=1, n \geq 0$. Therefore,

$$
\frac{\lambda}{N}\left(\left\|\frac{\left(f^{N}\right)^{*} \omega}{\mathrm{d}\left(f^{N}\right)}\right\|+\|\omega\|\right) \longrightarrow 0 \quad \text { as } N \rightarrow \infty .
$$

On the other hand, applying the first estimate of Part 2) of Lemma 3.1 yields that

$$
\begin{aligned}
& \frac{1}{N} \sum_{n=0}^{N-1} \frac{\left|\mathrm{d}\left(f^{n+1}\right)-\lambda \mathrm{d}\left(f^{n}\right)\right|}{\mathrm{d}\left(f^{n}\right)} \cdot\left\|\frac{\left(f^{n+1}\right)^{*} \omega}{\mathrm{d}\left(f^{n+1}\right)}\right\| \\
& \leq \frac{C}{N} \sum_{n=0}^{N-1} \frac{1}{n} \leq \frac{C \log N}{N} \rightarrow 0, \quad \text { as } N \rightarrow \infty .
\end{aligned}
$$

Inserting the last two estimates into the expression of $I I$, we obtain that $I I \rightarrow 0$ as $N \rightarrow \infty$. This, combined with (3.5) implies that

$$
f^{*} \sigma=\lambda \cdot \sigma+\mu[H]
$$

for some $\mu \in \mathbb{R}$. By equating the mass of both sides in the last equation and using (2.3), the lemma follows. that

$$
\Theta \circ F=\lambda \Theta+\frac{d-\lambda}{h} \log |H| .
$$

\section{Lemma 3.4.}

$$
\Theta \circ F_{n}= \begin{cases}\lambda^{n} \Theta+\frac{d-\lambda}{h} \cdot \sum_{j=1}^{n} \lambda^{j-1} \log \left|H \circ F_{n-j}\right|, & n=1, \ldots, n_{0}, \\ \lambda^{n} \Theta+\frac{d-\lambda}{h} \cdot \sum_{j=1}^{n_{0}} \lambda^{j-1} \log \left|H \circ F_{n-j}\right|, & n>n_{0} .\end{cases}
$$

Proof: We proceed by induction. For $n=1$ the above formula follows from (3.6). 
Suppose that the above inductive formula is true for $n$. We need to show it for $n+1$. Observe that

$$
\begin{aligned}
\Theta \circ F_{n+1}= & \Theta \circ F_{n} \circ F-\mathrm{d}\left(f^{n-n_{0}}\right) \log |H| \\
= & \lambda^{n} \Theta \circ F+\frac{d-\lambda}{h} \cdot \sum_{j=1}^{n_{0}} \lambda^{j-1} \log \left|H \circ F_{n-j} \circ F\right|-\mathrm{d}\left(f^{n-n_{0}}\right) \log |H| \\
= & \lambda^{n+1} \Theta+\frac{d-\lambda}{h} \cdot \lambda^{n} \log |H|-\mathrm{d}\left(f^{n-n_{0}}\right) \log |H| \\
& +\frac{d-\lambda}{h} \cdot \sum_{j=1}^{n_{0}} \lambda^{j-1} \log \left|H\left(H^{\mathrm{d}\left(f^{n-j-n_{0}}\right)} \cdot F_{n-j+1}\right)\right| \\
= & \lambda^{n+1} \Theta+\frac{d-\lambda}{h} \cdot \sum_{j=1}^{n_{0}} \lambda^{j-1} \log \left|H \circ F_{n-j+1}\right| \\
& +\left(\frac{d-\lambda}{h} \cdot \lambda^{n}+\frac{d-\lambda}{h} \cdot \sum_{j=1}^{n_{0}} \lambda^{j-1} h \mathrm{~d}\left(f^{n-j-n_{0}}\right)-\mathrm{d}\left(f^{n-n_{0}}\right)\right) \log |H| \\
& +\left(\lambda^{n-n_{0}}+\frac{d-\lambda}{h} \cdot \sum_{j=1}^{n_{0}} \lambda^{j-1} h \mathrm{~d}\left(f^{n-j-n_{0}}\right)-\mathrm{d}\left(f^{n-n_{0}}\right)\right) \log |H|, \\
= & \lambda^{n+1} \Theta+\frac{d-\lambda}{h} \cdot \sum_{j=1}^{n_{0}} \lambda^{j-1} \log \left|H \circ F_{n-j+1}\right| \\
& \left(\begin{array}{ll}
h \\
h
\end{array}\right)
\end{aligned}
$$

where the first equality follows from (3.3), the second one from the hypothesis of induction, the third one from (3.6) and (3.3), and the last one from (2.5). Therefore, the proof of the inductive formula will be complete for $n+1$ if one can show that for all $n \geq 0, S_{n}=0$, where

$$
S_{n}:=\lambda^{n}+(d-\lambda) \cdot \sum_{j=1}^{n_{0}} \lambda^{j-1} \mathrm{~d}\left(f^{n-j}\right)-\mathrm{d}\left(f^{n}\right) .
$$

It follows from (2.4)-(2.5) and the above formula for $S_{n}$ that $S_{n}-d S_{n-1}+$ $h S_{n-n_{0}-1}=0$ for all $n \geq n_{0}+1$. Hence, the proof will be complete if one can show that $S_{n}=0$ for $n=0, \ldots, n_{0}$. But the last assertion is equivalent to the identity

$$
\lambda^{n}+(d-\lambda) \cdot \sum_{j=1}^{n_{0}} \lambda^{j-1} \mathrm{~d}(f)^{n-j}=\mathrm{d}(f)^{n},
$$


which is clearly true by using the convention preceding Lemma 3.2. Hence, the proof is complete.

The following elementary lemma is needed.

Lemma 3.5. Let $(X, \mu)$ be a measurable space and $\left(g_{n}\right)_{n=0}^{\infty},\left(h_{n}\right)_{n=1}^{\infty} \subset$ $L^{1}(X, \mu)$ two sequence of complex-valued functions with $\left\|h_{n}\right\|_{L^{1}(X)} \leq 1$, $n \geq 1$. Let $P(t):=t^{n_{0}}+\alpha_{1} t^{n_{0}-1}+\cdots+\alpha_{n_{0}}$ be a polynomial whose roots are of modulus strictly smaller than 1 . Let $\left(\epsilon_{n}\right)_{n=n_{0}}^{\infty} \subset \mathbb{R}^{+}$be a sequence with $\lim _{n \rightarrow \infty} \epsilon_{n}=0$. Let $\left(\alpha_{1 n}\right)_{n_{0}}^{\infty}, \ldots,\left(\alpha_{n_{0} n}\right)_{n_{0}}^{\infty} \subset \mathbb{C}$ be $n_{0}$ sequences such that for all $n \geq n_{0}$ and $1 \leq j \leq n_{0}$,

- $g_{n}+\alpha_{1 n} g_{n-1}+\cdots+\alpha_{n_{0} n} g_{n-n_{0}}=h_{n}$;

- $\left|\alpha_{j n}-\alpha_{j}\right|<\epsilon_{n}$.

Then $\left(g_{n}\right)_{n=0}^{\infty}$ is bounded in $L^{1}(X, \mu)$.

Proof: Let $t_{1}, \ldots, t_{n_{0}}$ be the roots of $P(t)$. Consider two cases.

Case 1: $t_{1}, \ldots, t_{n_{0}}$ are distinct.

We can check that if $\gamma_{1}, \ldots, \gamma_{n_{0}} \in \mathbb{C}$ such that $\sum_{j=1}^{n_{0}} \gamma_{j} \cdot \frac{P(t)}{t-t_{j}} \equiv 0$ then $\gamma_{1}=\cdots=\gamma_{n_{0}}=0$. Consequently, there exist $\gamma_{1}, \ldots, \gamma_{n_{0}} \in \mathbb{C}$ such that

$$
\sum_{j=1}^{n_{0}} \gamma_{j} \cdot \frac{P(t)}{t-t_{j}} \equiv t^{n_{0}-1}
$$

Next, write

$$
\frac{P(t)}{t-t_{j}}=t^{n_{0}-1}+\beta_{j 1} t^{n_{0}-2}+\cdots+\beta_{j n_{0}-1}, \quad j=1, \ldots, n_{0} .
$$

Put

$$
f_{j n}:=g_{n}+\beta_{j 1} g_{n-1}+\cdots+\beta_{j n_{0}-1} g_{n-n_{0}+1}, \quad n \geq n_{0}-1,1 \leq j \leq n_{0} .
$$

Hence, (3.7) becomes

$$
\sum_{j=1}^{n_{0}} \gamma_{j} f_{j n}=g_{n-1}, \quad n \geq n_{0}-1,1 \leq j \leq n_{0} .
$$

The formula for $f_{j n}$, the first item of the hypothesis and identity (3.8) together imply that

$$
\left|f_{j n}-t_{j} f_{j, n-1}\right| \leq\left|h_{n}\right|+\epsilon_{n-1}\left|g_{n-1}\right|+\cdots+\epsilon_{n-n_{0}}\left|g_{n-n_{0}}\right|, \quad n \geq n_{0}, 1 \leq j \leq n_{0} .
$$


It follows from the last estimate and (3.9) that there is a finite positive constant $C$ such that

$$
\begin{array}{r}
M_{n}^{\prime} \leq \rho^{\prime} M_{n-1}^{\prime}+C\left(\epsilon_{n-1}+\cdots+\epsilon_{n-n_{0}}\right)\left(M_{n}^{\prime}+\cdots+M_{n-n_{0}+1}^{\prime}\right)+\left\|h_{n}\right\|_{L^{1}(X)}, \\
n \geq 2 n_{0},
\end{array}
$$

where $M_{n}^{\prime}:=\max \left\{\left\|f_{1 n}\right\|_{L^{1}(X)}, \ldots,\left\|f_{n_{0} n}\right\|_{L^{1}(X)}\right\}$ for all $n \geq n_{0}-1$, and $\rho^{\prime}:=\max _{1 \leq j \leq n_{0}}\left|t_{j}\right|$. Observe that $0<\rho^{\prime}<1$ since $\left|t_{j}\right|<1$.

Fix a constant $\rho: \rho^{\prime}<\rho<1$. Using the above estimate for $M_{n}^{\prime}$ repeatedly and taking into account that $\lim _{n \rightarrow \infty} \epsilon_{n}=0$, we may find a sufficiently large integer $N>n_{0}$ such that

$$
M_{n} \leq \rho M_{n-1}+2\left(\sum_{j=1}^{n_{0}}\left\|h_{n n_{0}-j}\right\|_{L^{1}(X)}\right), \quad n>N,
$$

where $M_{n}:=\max \left\{M_{n n_{0}-1}^{\prime}, \ldots, M_{n n_{0}-n_{0}}^{\prime}\right\}$ for all $n \geq N$. Consequently,

$$
M_{n} \leq \frac{2}{1-\rho} \cdot \sum_{k=0}^{n-N-1} \rho^{k}\left(\sum_{j=1}^{n_{0}}\left\|h_{(n-k) n_{0}-j}\right\|_{L^{1}(X)}\right)+\rho^{n-N} M_{N}
$$

for all $n \geq N$. This, combined with the hypothesis that $\left\|h_{n}\right\|_{L^{1}(X)} \leq 1$ for all $n \geq 1$, implies the existence of a finite positive constant $M$ such that

$$
\left\|f_{j n}\right\|_{L^{1}(X)}<M, \quad n \geq N, 1 \leq j \leq n_{0} .
$$

This, coupled with (3.9), gives the desired conclusion.

Case 2: $t_{1}, \ldots, t_{n_{0}}$ are not distinct.

Let $t_{1}, \ldots, t_{r}$ be all distinct roots of $P(t)$ with multiplicity $m_{1}, \ldots, m_{r}$ respectively. We can choose $\gamma_{11}, \ldots, \gamma_{1 m_{1}}, \ldots, \gamma_{r 1}, \ldots, \gamma_{r m_{r}} \in \mathbb{C}$ such that

$$
\sum_{j=1}^{r} \frac{\left(\gamma_{j 1}+\gamma_{j 2} t+\cdots+\gamma_{j m_{j}} t^{m_{j}-1}\right) P(t)}{\left(t-t_{j}\right)^{m_{j}}} \equiv t^{n_{0}-1}
$$

The remaining part of the proof follows along the same lines as in the previous case.

Let us recall that a quasi-plurisubharmonic function on $\mathbb{P}^{k}$ is an upper semi-continuous function $\phi: \mathbb{P}^{k} \rightarrow[-\infty, \infty)$ which is locally given as the sum of a plurisubharmonic and a smooth function. The following estimate due to V. Guedj (see Proposition 1.3 in [17]) is needed. 
Lemma 3.6. There exists a positive finite constant $C$ such that for all quasi-plurisubharmonic functions $\phi$ with $\max \phi=0, \mathrm{dd}^{\mathrm{c}} \phi \geq-\omega$, and for all $n \in \mathbb{N}$,

$$
\int_{\mathbb{P}^{k}}\left(|\phi| \circ f^{n}\right) \omega^{k} \leq C \sum_{j=0}^{n} \mathrm{~d}\left(f^{j}\right) .
$$

Now we arrive at

Proof of Part (i) of Main Theorem: For all $n>n_{0}$ consider the functions defined on $\mathbb{P}^{k}$

$$
h_{n}:=\Theta+\frac{d-\lambda}{h} \cdot \sum_{j=1}^{n_{0}} \frac{1}{\lambda^{n-j+1}} \log \left|H\left(\frac{F_{n-j}}{\left\|F_{n-j}\right\|}\right)\right|-\frac{1}{\lambda^{n}} \Theta\left(\frac{F_{n}}{\left\|F_{n}\right\|}\right) .
$$

By Lemma 3.4, we have that

$$
\frac{\log \left\|F_{n}\right\|}{\lambda^{n}}-\frac{d-\lambda}{\lambda} \cdot \sum_{j=1}^{n_{0}} \frac{\log \left\|F_{n-j}\right\|}{\lambda^{n-j}}=h_{n} .
$$

On the one hand, since $\lambda$ is a simple root of $P$ we know from Part 1) of Lemma 3.1 that $\mathrm{d}\left(f^{n}\right) \approx \lambda^{n}$. On the other hand, since $\Theta$ and $\log |H|$ are plurisubharmonic in $\mathbb{C}^{k+1}$, an application of Lemma 3.6 and the second estimate of Part 2) of Lemma 3.1 gives that $\left\|h_{n}\right\|_{L\left(\mathbb{P}^{k}, \omega^{k}\right)}<C$ for a finite constant $C$ independent of $n$. Moreover, the polynomial $t^{n_{0}}-\frac{d-\lambda}{\lambda}$. $\sum_{j=1}^{n_{0}} t^{n_{0}-j}$ is equal to $\frac{1}{\lambda^{n_{0}}} \frac{P(\lambda t)}{\lambda t-\lambda}$ by using the identity $P(\lambda t)=P(\lambda t)-P(\lambda)$. Therefore, by (2.5) and by the hypothesis that $\lambda$ is a simple root of $P$, we see that all roots of $t^{n_{0}}-\frac{d-\lambda}{\lambda} \cdot \sum_{j=1}^{n_{0}} t^{n_{0}-j}$ are of modulus strictly smaller than 1 . Hence, we are in the position to apply Lemma 3.5 to the relations (3.10) with $\alpha_{j}:=-\frac{d-\lambda}{\lambda}$ and $\alpha_{j n}:=-\frac{d-\lambda}{\lambda}, 1 \leq j \leq n_{0}$. Consequently, it follows that $\frac{\log \left\|F_{n}\right\|}{\lambda^{n}}$ is locally uniformly bounded in $L^{1}\left(\mathbb{C}^{k+1}\right)$-norm. This proves Part (i).

Proof of Part (ii): Using identity (3.3) we have

$$
\begin{aligned}
\frac{\log \left\|F_{n} \circ F\right\|}{\mathrm{d}\left(f^{n}\right)}= & \frac{\log \left\|F_{n+1}\right\|}{\mathrm{d}\left(f^{n}\right)}+\frac{\mathrm{d}\left(f^{n-n_{0}}\right) \log |H|}{\mathrm{d}\left(f^{n}\right)} \\
= & \lambda \cdot \frac{\log \left\|F_{n+1}\right\|}{\mathrm{d}\left(f^{n+1}\right)} \\
& +\left(\frac{\mathrm{d}\left(f^{n+1}\right)}{\mathrm{d}\left(f^{n}\right)}-\lambda\right) \frac{\log \left\|F_{n+1}\right\|}{\mathrm{d}\left(f^{n+1}\right)}+\frac{\mathrm{d}\left(f^{n-n_{0}}\right) \log |H|}{\mathrm{d}\left(f^{n}\right)} .
\end{aligned}
$$


Now take the (limsup)* of both sides of the above identity. By Part (i), the left hand side is then $u \circ F$ and the first term of the right hand side is $\lambda \cdot u$. The second term of the right hand side is 0 by using the first estimate of Part 2) of Lemma 3.1 and the fact already proved in Part (i) that $\frac{\log \left\|F_{n}\right\|}{\mathrm{d}\left(f^{n}\right)}$ is locally uniformly bounded in $L^{1}\left(\mathbb{C}^{k+1}\right)$-norm. The last term of the right hand side converges to $\frac{1}{\lambda^{n} 0} \cdot \log |H|$ using Part 1) of Lemma 3.1: $\mathrm{d}\left(f^{n}\right) \approx \lambda^{n}$. Summarizing, we have shown that

$$
u \circ F=\lambda \cdot u+\frac{1}{\lambda^{n_{0}}} \cdot \log |H|=\lambda \cdot u+\frac{d-\lambda}{h} \cdot \log |H|,
$$

where the last equality follows from equation (2.5). This proves (ii).

Proof of Part (iii): Let $p \in U$, where $U$ is an open set contained in the Fatou set. Shrinking $U$ if necessary, we may assume that a subsequence $f^{n_{j}}$ converges in $U$ to a holomorphic map $h$ and that $f^{n_{j}}(U) \subset$ $\left\{z_{0}=1,\left|z_{j}\right|<2\right\}$. We can then write

$$
\frac{\log \left\|F_{n_{j}}\right\|}{\mathrm{d}\left(f^{n_{j}}\right)}=\frac{\log \left\|\left(F_{n_{j}}\right)_{0}\right\|}{\mathrm{d}\left(f^{n_{j}}\right)}+\frac{1}{\mathrm{~d}\left(f^{n_{j}}\right)} \log \|\left(1, A_{j}^{1}, \ldots, A_{j}^{k} \| .\right.
$$

The last term converges uniformly to 0 , and the first term is pluriharmonic. Hence, using Part (i) the function $u$ is pluriharmonic on $U$, and $U$ does not intersect the support of $T$.

\section{Examples}

First we recall the result from our previous work [19].

4.1. A sufficient condition for QAS self-maps. In [16] Fornæss and Sibony give the following definition.

Definition 4.1. A hypersurface $\mathcal{H} \subset \mathbb{P}^{k}$ is said to be a degree lowering hypersurface of $f$ if, for some (smallest) $n \geq 1, f^{n}(\mathcal{H}) \subset \mathcal{I}(f)$. The integer $n$ is then called the height of $\mathcal{H}$.

The following (see Proposition 3.2 in $[\mathbf{1 9}]$ ) gives us the structure of a non AS self-map.

Proposition 4.2. Let $f$ be a meromorphic self-map of $\mathbb{P}^{k}$. Then there is exactly an integer $M \geq 0, M$ degree lowering hypersurfaces $\mathcal{H}_{j}$ with height $n_{j}, j=1, \ldots, M$, satisfying the following properties:

(i) All the numbers $n_{j}, j=1, \ldots, M$, are distinct.

(ii) $\operatorname{codim}\left(f^{m}\left(\mathcal{H}_{j}\right)\right)>1$ for $m=1, \ldots, n_{j}$, and $j=1, \ldots, M$. 
(iii) For any degree lowering irreducible hypersurface $\mathcal{H}$ of $f$, there are integers $n \geq 0$ and $1 \leq j \leq M$ such that $f^{n}(\mathcal{H})$ is a hypersurface and $f^{n}(\mathcal{H}) \subset \mathcal{H}_{j}$.

So $f$ is AS if and only if $M=0$.

Definition 4.3. Under the hypothesis and the notation of Proposition 4.2 , for every $j=1, \ldots, M, \mathcal{H}_{j}$ is said to be the primitive degree lowering hypersurface of $f$ with the height $n_{j}$.

We are now able to state a sufficient criterion for QAS maps (see Main Theorem in [19]).

Theorem 4.4. A meromorphic self-map $f$ of $\mathbb{P}^{k}$ is $Q A S$ if it satisfies the following properties (i)-(iii):

(i) There is only one primitive degree lowering hypersurface, in other words, $M=1$.

(ii) Let $\mathcal{H}_{0}$ be the hypersurface from Part (i) and let $n_{0}$ be its height. Then for every irreducible component $\mathcal{H}$ of $\mathcal{H}_{0}$ and every $m=$ $1, \ldots, n_{0}, f^{m}(\mathcal{H}) \not \subset \mathcal{H}_{0}$.

(iii) For every irreducible component $\mathcal{H}$ of $\mathcal{H}_{0}$, one of the following two conditions holds

(iii) $1 \quad f^{m}(\mathcal{H}) \not \subset \mathcal{I}(f)$ for all $m \geq n_{0}+1$,

(iii) 2 there is an $m_{0} \geq n_{0}$ such that $f^{m_{0}+1}(\mathcal{H})$ is a hypersurface and $f^{m}(\mathcal{H}) \not \subset \mathcal{I}(f)$ for all $m$ verifying $n_{0}+1 \leq m \leq m_{0}$.

Theorem 4.4 coupled with Proposition 4.2 gives us an efficient and simple method to check if a map is QAS. The remaining of this section is devoted to the study of new parameterized families of QAS maps in $\mathbb{P}^{2}$.

4.2. New family of QAS self-maps of $\mathbb{P}^{\mathbf{2}}$. Let $P$ be a (not necessarily irreducible) homogeneous polynomial in $\mathbb{C}^{3}$. Let $Q_{1}, Q_{2}$ and $Q_{3}$ be (not necessarily irreducible) homogeneous polynomials in $\mathbb{C}^{3}$ of the same degree. Let $R$ be a (not necessarily irreducible) homogeneous polynomial in $\mathbb{C}^{3}$ such that $\operatorname{deg}(R)=\operatorname{deg}(P)+\operatorname{deg}\left(Q_{1}\right)$ and that

$$
P(1,1,1) Q_{j}(1,1,1)=R(1,1,1) \neq 0, \quad j=1,2,3 .
$$

Suppose for the moment that $P Q_{1}-R, P Q_{2}-R, P Q_{3}-R$ have no nontrivial common factor, we are able to define a dominant meromorphic map of $\mathbb{P}^{2}$

$$
f([z: w: t]):=\left[P Q_{1}-R: P Q_{2}-R: P Q_{3}-R\right] .
$$


It can be checked that for every $(a, b, c) \in \mathbb{C}^{3} \backslash\{0\}$ with $a+b+c=0$, the hypersurface $\left\{a Q_{1}+b Q_{2}+c Q_{3}=0\right\}$ is sent by $f$ into the complex line $\{a z+b w+c t=0\}$.

Proposition 4.5. Suppose that for all $(a, b, c) \in \mathbb{C}^{3} \backslash\{0\}$ with $a+b+c=$ 0 , every irreducible component of the hypersurface $\left\{a Q_{1}+b Q_{2}+c Q_{3}\right\}$ is sent by $f$ onto a hypersurface. Suppose in addition that every irreducible component of the hypersurface $\{P=0\}$ is sent by $f^{2}$ onto a hypersurface and that if $\mathcal{G}$ is an irreducible hypersurface such that $f(\mathcal{G})=[1: 1: 1]$ then $\mathcal{G} \subset\{P=0\}$. Then $f$ satisfies the properties (i)-(iii) listed in Theorem 4.4, in particular, $f$ is $Q A S$.

Proof: First observe by (4.2) and (4.1) that the hypersurface $\{P=0\}$ is sent by $f$ to the point $[1: 1: 1] \in \mathcal{I}(f)$. We will show that there is no irreducible degree lowering hypersurface other than the components of $\{P=0\}$. To do this suppose, in order to get a contradiction, that $G$ is an irreducible homogeneous polynomial in $\mathbb{C}^{3}$ such that $\mathcal{G} \not \subset\{P=0\}$ and that $f(\mathcal{G})$ is a point $[a: b: c] \in \mathbb{P}^{2}$, where $\mathcal{G}$ is the hypersurface $\{G=$ $0\}$ in $\mathbb{P}^{2}$. Suppose, without loss of generality, that $a \neq 0$. We deduce from (4.2) and the equality $f(\mathcal{G})=[a: b: c]$ that $G$ divides both polynomials $P\left(b Q_{1}-a Q_{2}\right)-(b-a) R$ and $P\left(c Q_{1}-a Q_{3}\right)-(c-a) R$. Hence, $G$ divides the polynomial

$$
P \cdot\left((c-a)\left(b Q_{1}-a Q_{2}\right)-(b-a)\left(c Q_{1}-a Q_{3}\right)\right) .
$$

Since $\mathcal{G} \not \subset\{P=0\}$ and $G$ is irreducible, we see that $G$ divides the polynomial $(a c-a b) Q_{1}+\left(a^{2}-a c\right) Q_{2}+\left(a b-a^{2}\right) Q_{3}$. Since $a \neq 0$, we deduce from the first hypothesis that either $f(\mathcal{G})$ is a hypersurface or $a=b=c$. The former case contradicts the assumption that $f(\mathcal{G})$ is a point $[a: b: c] \in \mathbb{P}^{2}$. The latter case implies that $f(\mathcal{G})=[1: 1: 1]$, which, by the third hypothesis, gives that $\mathcal{G} \subset\{P=0\}$, which contradicts our assumption.

We have shown that $\{P=0\}$ is the unique primitive degree lowering hypersurface and its height is 1 . Since by (4.1) $[1: 1: 1] \notin\{P=0\}$, and every irreducible component of the hypersurface $\{P=0\}$ is sent by $f^{2}$ onto a hypersurface, it follows that $f$ satisfies (i)-(ii)-(iii) $)_{2}$ of Theorem 4.4 .

Now we will discuss cases when the hypotheses of Proposition 4.5 are fulfilled. 
Corollary 4.6. Suppose that $Q_{2}-Q_{1}, Q_{3}-Q_{1}$ are coprime and that $P, R$ are coprime. Then $P Q_{1}-R, P Q_{2}-R, P Q_{3}-R$ have no nontrivial common factor. Moreover, for every irreducible hypersurface $\mathcal{G}$ with $f(\mathcal{G})=[1: 1: 1]$, we have $\mathcal{G} \subset\{P=0\}$. Here $f$ is defined by (4.2).

Proof: It is left to the interested reader as an exercise.

Corollary 4.7. Suppose that the pre-image of the point $[1: 1: 1]$ by the map $\mathbb{P}^{2} \ni[z: w: t] \mapsto\left[Q_{1}: Q_{2}: Q_{3}\right]$ is a set of finite points and that for every $[z: w: t] \in\{P=0\} \cap\{R=0\}$ and every $(a, b, c) \in \mathbb{C}^{3} \backslash\{0\}$ with $a+b+c=0$, we have $\left(a Q_{1}+b Q_{2}+c Q_{3}\right)(z, w, t) \neq 0$. Suppose in addition that for every $(a, b, c) \in \mathbb{C}^{3} \backslash\{0\}$ with $a+b+c=0$, two polynomials $P$ and $a Q_{1}+b Q_{2}+c Q_{3}$ are coprime. Then every irreducible component of the hypersurface $\left\{a Q_{1}+b Q_{2}+c Q_{3}\right\}$ is sent by $f$ onto a hypersurface.

Proof: In order to get a contradiction, suppose that $\mathcal{G}$ is an irreducible component of the hypersurface $\left\{a Q_{1}+b Q_{2}+c Q_{3}\right\}$ and $f(\mathcal{G})$ is a point $p \in$ $\mathbb{P}^{2}$, where $(a, b, c) \in \mathbb{C}^{3} \backslash\{0\}$ with $a+b+c=0$. Using the explicit formula (4.2), the second hypothesis ensures that there exists

$$
\left[z_{0}: w_{0}: t_{0}\right] \in\left(\left\{a Q_{1}+b Q_{2}+c Q_{3}\right\} \cap\{P=0\}\right) \backslash \mathcal{I}(f) .
$$

Consequently, we get $p=f\left(\left[z_{0}: w_{0}: t_{0}\right]\right)=[1: 1: 1]$. This implies that either the map $\mathbb{P}^{2} \ni[z: w: t] \mapsto\left[Q_{1}: Q_{2}: Q_{3}\right]$ sends $\mathcal{G}$ to the point $[1: 1: 1]$ or $\mathcal{G} \subset\{P=0\}$. But the former case contradicts the first hypothesis whereas the latter case contradicts the third hypothesis.

Corollary 4.8. Suppose that for every $(a, b, c) \in \mathbb{C}^{3} \backslash\{0\}$, two polynomials $P$ and $a Q_{1}+b Q_{2}+c Q_{3}$ are coprime. Suppose in addition that the $3 \times 3$ matrix whose $j$-th line is

$$
\left(\frac{\partial\left(P Q_{j}-R\right)}{\partial z}(1,1,1) \quad \frac{\partial\left(P Q_{j}-R\right)}{\partial w}(1,1,1) \quad \frac{\partial\left(P Q_{j}-R\right)}{\partial t}(1,1,1)\right)
$$

has the rank $\geq 2$. Then every irreducible component of the hypersurface $\{P=0\}$ is sent by $f^{2}$ onto a hypersurface.

Proof: Let $F: \mathbb{C}^{3} \rightarrow \mathbb{C}^{3}$ be given by

$$
F=\left(F_{1}, F_{2}, F_{3}\right):=\left(P Q_{1}-R, P Q_{2}-R, P Q_{3}-R\right) .
$$

Then a straightforward computation shows that the $j$-th component of $\frac{F \circ F}{P}(1 \leq j \leq 3)$ has the form

$\frac{\partial\left(P Q_{j}-R\right)}{\partial z}(F) \cdot Q_{1}+\frac{\partial\left(P Q_{j}-R\right)}{\partial w}(F) \cdot Q_{2}+\frac{\partial\left(P Q_{j}-R\right)}{\partial t}(F) \cdot Q_{3}+\mathcal{O}(P)$,

where $\mathcal{O}(P)$ is a polynomial which can be factored by $P$. Observe that the proof of the corollary will be complete if we can show that for any 
fixed irreducible divisor $S$ of $P$, the image of $[S=0]$ by $\frac{F \circ F}{P}(1 \leq j \leq 3)$ is a curve. Using the above formula, this task is reduced to show that the (not necessarily dominant) rational map of $\mathbb{P}^{2}$ whose $j$-th component is

$$
\begin{array}{r}
\frac{\partial\left(P Q_{j}-R\right)}{\partial z}(1,1,1) \cdot Q_{1} \\
+\frac{\partial\left(P Q_{j}-R\right)}{\partial w}(1,1,1) \cdot Q_{2} \\
+\frac{\partial\left(P Q_{j}-R\right)}{\partial t}(1,1,1) \cdot Q_{3}
\end{array}
$$

does not map $[S=0]$ to a point. But this is always satisfied taking into account the hypothesis.

Now we fix the degrees of $P$ and $Q_{1}$. Using the above corollaries, we see easily that with a generic choice of the coefficients of $R, P, Q_{1}, Q_{2}$, $Q_{3}$ such that relation (4.1) holds, the hypotheses of Corollaries 4.6, 4.7 and 4.8 are fulfilled. We thus obtain a family of non AS but QAS selfmaps. The characteristic polynomial of maps in this family is (see (2.5))

$$
P(t):=t^{2}-\left(\operatorname{deg}(P)+\operatorname{deg}\left(Q_{1}\right)\right) t+\operatorname{deg}(P) .
$$

\section{References}

[1] E. Bedford And K. Kim, Periodicities in linear fractional recurrences: degree growth of birational surface maps, Michigan Math. J. 54(3) (2006), 647-670. DOI: $10.1307 / \mathrm{mmj} / 1163789919$.

[2] E. Bedford AND K. KIM, Degree growth of matrix inversion: birational maps of symmetric, cyclic matrices, Discrete Contin. Dyn. Syst. 21(4) (2008), 977-1013. DOI: 10.3934/dcds.2008.21.977.

[3] A. M. Bonifant, Degrees of non-linearity in higher dimensional complex dynamics, Thesis, CINVESTAV-IPN, Mexico (1997).

[4] A. M. Bonifant And J. E. Fornæss, Growth of degree for iterates of rational maps in several variables, Indiana Univ. Math. J. 49(2) (2000), 751-778. DOI: 10.1215/10.1512/iumj .2000.49.1813.

[5] S. Boucksom, Ch. Favre, And M. Jonsson, Degree growth of meromorphic surface maps, Duke Math. J. 141(3) (2008), 519-538. DOI : $10.1215 / 00127094-2007-004$.

[6] J. Diller AND CH. FAvre, Dynamics of bimeromorphic maps of surfaces, Amer. J. Math. 123(6) (2001), 1135-1169. DOI: 10.1353/ajm.2001.0038. 
[7] T.-C. Dinh, R. Dujardin, And N. Sibony, On the dynamics near infinity of some polynomial mappings in $\mathbb{C}^{2}$, Math. Ann. 333(4) (2005), 703-739. DOI: 10.1007/s00208-005-0661-3.

[8] T.-C. Dinh and N. Sibony, Dynamique des applications polynomiales semi-régulières, Ark. Mat. 42(1) (2004), 61-85.

[9] T.-C. Dinh And N. Sibony, Regularization of currents and entropy, Ann. Sci. École Norm. Sup. (4) 37(6) (2004), 959-971. DOI: 10.1016/j . ansens. 2004.09.002.

[10] T.-C. Dinh And N. Sibony, Green currents for holomorphic automorphisms of compact Kähler manifolds, J. Amer. Math. Soc. 18(2) (2005), 291-312 (electronic). DOI: 10.1090/S0894-0347-0400474-6.

[11] T.-C. Dinh And N. Sibony, Une borne supérieure pour l'entropie topologique d'une application rationnelle, Ann. of Math. (2) 161(3) (2005), 1637-1644. DOI: 10.4007/annals.2005.161.1637.

[12] T.-C. Dinh And N. Sibony, Dynamics in several complex variables: endomorphisms of projective spaces and polynomial-like mappings, in: "Holomorphic dynamical systems", Lecture Notes in Math. 1998, Springer, Berlin, 2010, pp. 165-294. DOI : 10.1007/9783-642-13171-4_4.

[13] Ch. Favre and M. Jonsson, Eigenvaluations, Ann. Sci. École Norm. Sup. (4) 40(2) (2007), 309-349. DOI: 10.1016/j.ansens. 2007.01 .002 .

[14] Ch. Favre and M. Jonsson, Dynamical compactifications of $\mathbf{C}^{2}$, Ann. of Math. (2) 173(1) (2011), 211-248. DOI: 10.4007/annals. 2011.173.1.6.

[15] J. E. Fornæess and N. Sibony, Complex dynamics in higher dimensions, Notes partially written by Estela A. Gavosto, in: "Complex potential theory" (Montreal, PQ, 1993), NATO Adv. Sci. Inst. Ser. C Math. Phys. Sci. 439, Kluwer Acad. Publ., Dordrecht, 1994, pp. 131-186.

[16] J. E. Fornæess And N. Sibony, Complex dynamics in higher dimension. II, in: "Modern methods in complex analysis" (Princeton, NJ, 1992), Ann. of Math. Stud. 137, Princeton Univ. Press, Princeton, NJ, 1995, pp. 135-182.

[17] V. Guedu, Decay of volumes under iteration of meromorphic mappings, Ann. Inst. Fourier (Grenoble) 54(7) (2004), 2369-2386 (2005). DOI: 10.5802/aif.2083. 
[18] B. Hasselblatt and J. Propp, Degree-growth of monomial maps, Ergodic Theory Dynam. Systems 27(5) (2007), 1375-1397. DOI : $10.1017 / \mathrm{S} 0143385707000168$.

[19] V.-A. NGUYÊN, Algebraic degrees for iterates of meromorphic selfmaps of $\mathbb{P}^{k}$, Publ. Mat. 50(2) (2006), 457-473. DOI: 10.5565/ PUBLMAT_50206_10.

[20] N. Sibony, Dynamique des applications rationnelles de $\mathbb{P}^{k}$, in: " $D y$ namique et géométrie complexes" (Lyon, 1997), Panor. Synthèses 8 , Soc. Math. France, Paris, 1999, pp. ix-x, xi-xii, 97-185.

Mathématique-Bâtiment 425

UMR 8628, 15 rue Georges Clémenceau

Université Paris-Sud

F-91405 Orsay

France

E-mail address: VietAnh.Nguyen@math.u-psud.fr

URL: http://www.math.u-psud.fr/ vietanh/

Primera versió rebuda el 9 de desembre de 2010, darrera versió rebuda el 5 d'abril de 2011. 